\title{
The Numerical Simulation of Characteristics of Tidal Wave Propagation in Yangtze Estuary
}

\author{
Lu Chuanteng ${ }^{1, a}$, Luo Xiaofeng ${ }^{1, b}$ \\ ${ }^{1}$ Nanjing Hydraulic Research Institute, Nanjing, Jiangsu, China \\ alct000abc@163.com, ${ }^{b}$ cjk3d@126.com
}

\begin{abstract}
Keywords: Yangtze estuary; Tidal wave propagation; Numerical simulation; Harmonic constants Abstract. Using the finite volume method, a 2D tidal mathematic model from Datong to open sea is developed to study the Yangtze estuary. On the basis of good verification, the characteristics of tidal wave propagation, such as tidal level, tidal range, harmonic constants, the relationship between tidal level and tidal current etc., are analyzed in Yangtze estuary using the mathematical model. The research indicates that semidiurnal component tide is dominant in Yangtze estuary, and the changing of M2 amplitude coincides well with the changing of tidal range. The property of advancing wave is strengthened gradually from Niupijiao station to tidal current limit(near Jiangyin station).
\end{abstract}

\section{Introduction}

There are three dividing points and four channels that drain to near-shore bathing waters, and the bed configuration is complicated and volatile in Yangtze estuary. The runoff and tide are the main hydrodynamic force which interact over time and distance. The tidal power takes the form of tidal wave propagation which is of great significance to shipping, flood control, industrial and agricultural production in river.

Scholars at home and abroad have carried out much research on tidal wave propagation in Yangtze estuary. Yu DongSheng[1] believes that Coriolis force makes the tidal wave distortion in northern bank heavier than that in southern bank(except for north branch) in Yangtze estuary, and it is the main causing of estuarine circulation. Lei Zhiyi[2] thinks that the runoff power is dominant above Jiangyin, while tide power becomes stronger below Jiangyin. After analyzing the tidal level vatiation features below Xuliujing in Yangtze estuary, Xu Hanxing[3] regards "cap in sleeve" of water level as a normal phenomenon in tidal wave propagation. Chen Zhichang[4] is the first one using physical model to simulate Coriolis force to improve the accuracy of tidal wave propagation in experiment. Shen Huanting[5] discussed the tidal wave propagation in Yangtze estuary below Xuliujing. Yan Yixin[6] believes that the average tidal level in north passage changes few, whereas the tidal range changes much, after the Yangtze estuary Deepwater Channel Regulation Project. Yu DongSheng[7] points that there are lateral, vertical and horizontal circulation in Yangtze estuary based on analyzing the ADCP hydrological data. Yang Zhengdong[8] thinks that the tide in north branch is regular semidiurnal tide, while it is irregular semidiurnal tide in south branch, north channel and south channel. What's more, the tidal deformation is the heaviest at YonglongSha in north branch.

The researches above in Yangtze estuary generally represent the current studying status on tidal wave propagation. The researches on tidal wave propagation are mostly focused on the river below Jiangyin, while there are rare studies on the whole river of Yangtze estuary. To have a better understand about tidal wave propagation in Yangtze estuary, it is essential to perform a study on the river above Jiangyin. On the basis of previous studies, a 2D tidal mathematic model from Datong to open sea is developed to study the characteristics of tidal wave in Yangtze estuarine, making the tidal wave propagation theory complementary and improved. 


\section{Establishment of the mathematic model In Yangtze estuary}

\section{Governing Equation}

The 2D shallow water current and sediment equations written in conversation and vector form are:

$$
\frac{\partial \mathrm{U}}{\partial \mathrm{t}}+\nabla \mathrm{E}=\mathrm{M}+\nabla \mathrm{E}^{\mathrm{d}}, \text { where: } \mathrm{U}=(\mathrm{H}, \mathrm{Hu}, \mathrm{Hv})^{\mathrm{T}}
$$

$E=(F, G)$, Where: $F=\left(\begin{array}{l}H u \\ H u^{2}+g H^{2} / 2 \\ H u v\end{array}\right), G=\left(\begin{array}{l}H v \\ H u v \\ H v^{2}+g H^{2} / 2\end{array}\right), E^{d}=\left(F^{d}, G^{d}\right)$, where: $F^{d}=\left(\begin{array}{l}0 \\ \varepsilon_{x} H \partial u \\ \varepsilon_{x} H \partial v\end{array}\right.$

$\mathrm{H}-\operatorname{Depth}(\mathrm{m}) ; \mathrm{u} 、 \mathrm{v}-\mathrm{The} \mathrm{x} 、 \mathrm{y}$ components of velocity $\mathrm{V}(\mathrm{m} / \mathrm{s}) ; \mathrm{t}$-Time(s);

$\mathrm{f}$-Coriolis coefficient; $\mathrm{g}$ - Gravitational acceleration $(\mathrm{m} / \mathrm{s} 2) ; \mathrm{N}_{\mathrm{x}} 、 \mathrm{~N}_{\mathrm{y}}$ - Turbulence viscosity coefficient in $\mathrm{x} 、 \mathrm{y}$ direction $(\mathrm{m} 2 / \mathrm{s}) . ; M_{0 x} 、 M_{0 y}-$ Variation of bed elevation in $\mathrm{x} 、 \mathrm{y}$ direction; $\mathrm{M}_{\mathrm{fx}} 、 \mathrm{M}_{\mathrm{fy}}$-Bottom friction in $\mathrm{X} 、 \mathrm{y}$ direction;

The element of $\mathrm{i}$ can be denoted as $\Omega_{\mathrm{i}}$ where the control equations can be integrated. Applying the Gauss theorem, Eq.(1) can be integrated over an arbitrary control volume V as:

$$
\frac{\partial}{\partial t} \int_{\Omega_{1}} U d \Omega_{\mathrm{i}}+\oint_{\partial \Omega_{4}}\left(E \cdot \bar{n}_{\mathrm{i}}-E^{d} \cdot \bar{n}_{\mathrm{i}}\right) \mathrm{dl}=\int_{\Omega_{\mathrm{i}}} \mathrm{Md} \Omega_{\mathrm{i}}
$$

Using finite volume method to solve the Eq.(2). There are three parts in Eq.(2), which are convection term, turbulent term and bottom slope term. The idiographic process can refer to reference [9].

\section{The Range of Mathematic Model}

The tidal limit of Yangtze River is located in Datong, Anhui province, where is chosen to be the upper boundary of the model. The lower boundary is located in -50 contour in open sea where the tidal wave is not be influenced by runoff. The length of the model is over $700 \mathrm{KM}$. The northern boundary is to LvSi Harbor in Jiang province, and the southern boundary is to JinShanZui in ZheJiang province. The width of model is over $150 \mathrm{KM}$, as Fig.1.

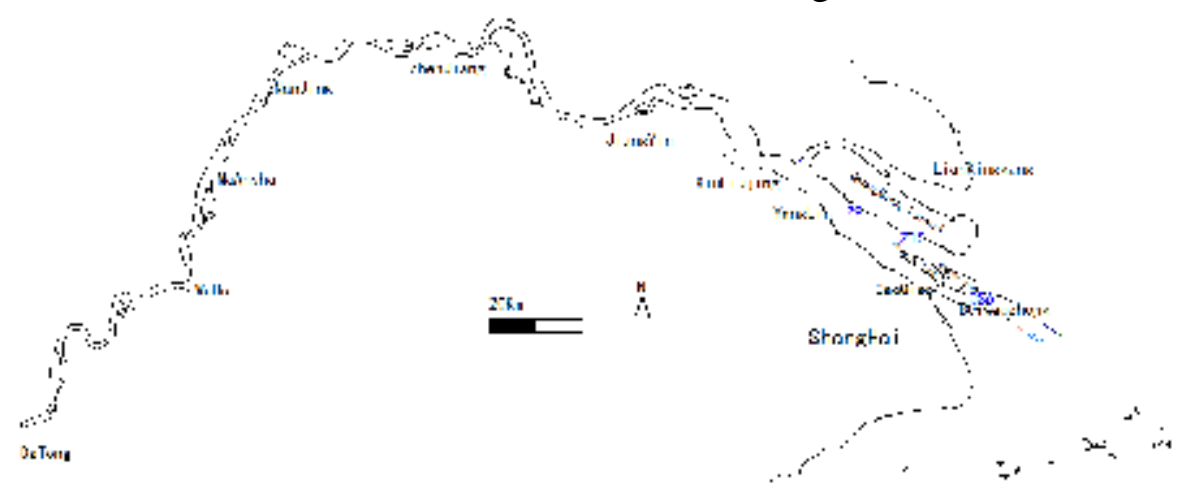

Fig.1 Range of the Mathematic Model 


\section{Calculation Parameters}

Tab. 1 is calculation parameters of the mathematic model in this paper.

Tab.1 Calculation parameters of the mathematic model

\begin{tabular}{cc}
\hline Parameter names & Parameter values \\
\hline Minimum side length of elements & $128 \mathrm{~m}$ \\
Number of elements & 114489 \\
Time step & $4 \mathrm{~s}$ \\
Roughness coefficient & $0.03 \sim 0.012$ (Linear interpolation from Datong to \\
Eddy viscosity coefficient & open sea) \\
Moving boundary depth & 0.1 \\
\end{tabular}

\section{Model Validation}

The calculation interval of mathematical model is from August 12th 2005 to September 26th 2005 for 15 days. Due to the measured hydrological time inconsistency, using simultaneous observation data from August 18th 2005 to September 25th 2005 as the verification data, and the verification points are shown in Fig.1. The average runoff during validation in Datong is $41485.7 \mathrm{~m} 3 / \mathrm{s}$. The verification results of tidal level and tidal current are shown in Fig. $2 \sim 3$ respectively. The verification results indicate that the calculated tidal level and tidal current of most verification points coincide better with the measured values, and the relative errors are within $10 \%$. With the better verification, the mathematical model can simulate the tidal wave propagation in Yangtze River.
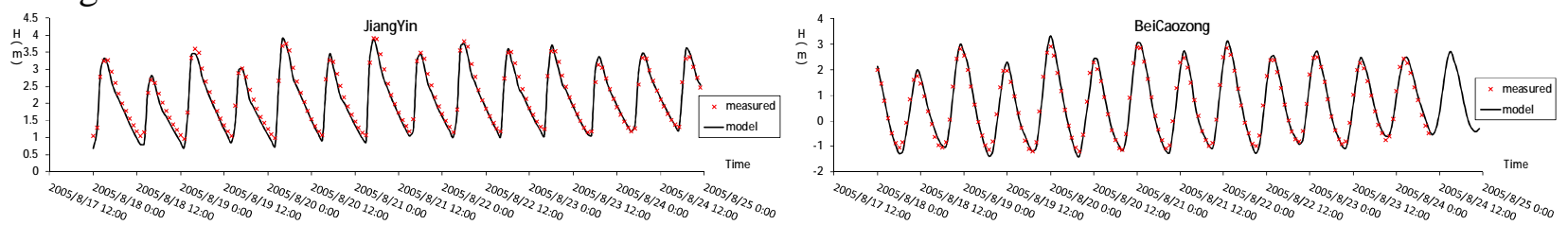

Fig. 2 Tidal level Verification
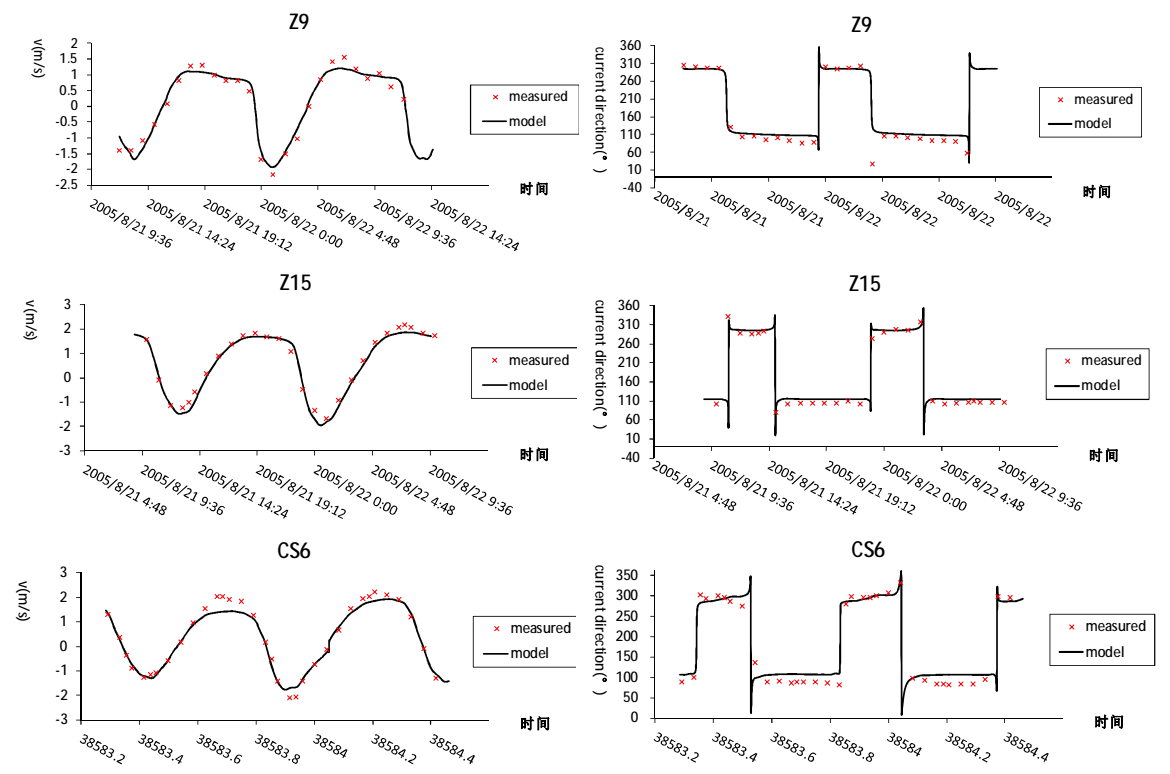

Fig.3 Tidal Current Verification 


\section{The Characteristics of tidal wave propagating in Yangtze estuary}

\section{Tidal Level}

Fig. 4 is the changing of tidal level along the river in Yangtze estuary. For the tidal wave energy accumulation causing by the boundary shape contraction of Yangtze estuary and the tidal wave refection causing by Yangtze estuary Deepwater Channel Regulation Project in the nearby Niupijiao station, the high tidal level gets higher during the tidal wave propagation from open sea to upstream, while low tidal level gets lower. In the river from Niupijiao to Sima(North of Yangzhong), the high tidal level and low tidal level get higher as a result of runoff resistance and bed configuration, and low tidal level surface slope is significantly greater than the high tide for the shallow water depth in low tide. The river from Sima to Zhenjiang is one of the most complex terrain in Yangtze estuary, and there are many river bends and scattered shoals in the channel. Both of high tidal level and low tidal level get higher visibly when tidal wave arrive here, besides, low tidal level arises much more than high tidal level. In the channel above Zhenjiang, for the weaker tidal wave energy, high tidal level approaches to low tidal level gradually, and there is little difference when the tidal wave propagates to Wuhu. Average tidal level gets higher for open sea to upstream, and the average tidal level surface slope in the channel above Sima is sharper than that below Sima.

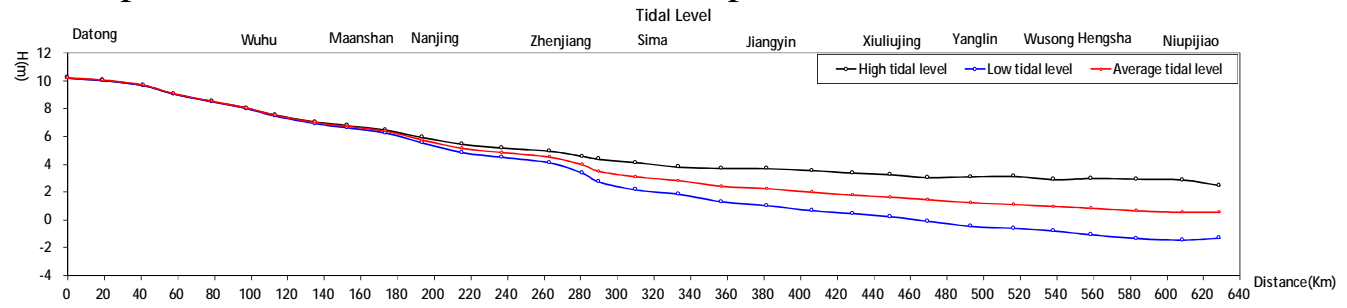

Fig.4 The Tidal level in Yangtze Estuary

\section{Tidal Range}

Fig.5 shows the tidal range in Yangtze estuary. From open sea to upstream, tidal range gets larger firstly, and then goes down. Similar to tidal level, for the tidal wave energy accumulation causing by the boundary shape contraction of Yangtze estuary and the tidal wave refection causing by Yangtze estuary Deepwater Channel Regulation Project, tidal range reaches to its largest, about $4.3 \mathrm{~m}$, in the nearby Niupijiao station. As tidal wave spreads to upstream, tidal wave energy goes weaker as a result of the runoff resistance and riverbed bottom friction. When tidal wave spreads to Sima, tidal range decreases to about $2 \mathrm{~m}$. The main river above Sima turns over $90^{\circ}$, and tidal wave may happen reflecting, so the tidal range increases slightly. There are many river bends and scattered shoals in the river from Sima to Zhenjiang where tidal range falls quickly, and the tidal range is about $1.1 \mathrm{~m}$ at Zhenjiang. In the river above Zhenjiang, the tidal range falls slowly for the tidal wave energy weakening. When the tidal wave spreads to Wuhu, the tidal range is less than $0.1 \mathrm{~m}$.

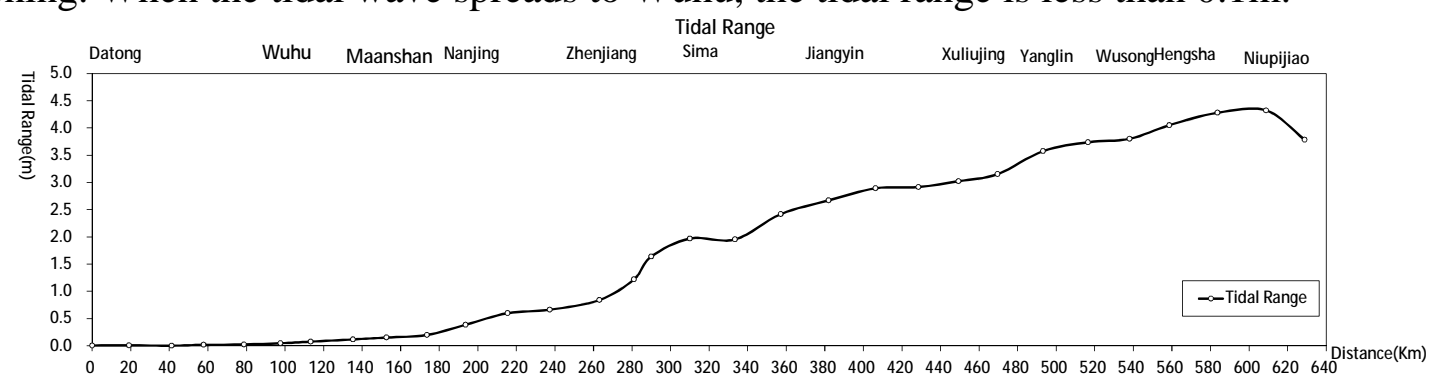

Fig.5 The tidal range in Yangtze estuary 


\section{Harmonic Constants}

Using O1, K1, M2, S2, M4 and MS4 component tides to compute harmonic constants based on mathematic model calculations for 15 days.

Amplitude of M2 is dominant in those six basic component tides. From Fig.6, the changing of M2 amplitude corresponds to the changing of tidal range, that is to say, M2 component tide plays a decisive role in Yangtze estuary tidal wave propagation. Taking K1 component tide as the respective of diurnal component tide, and it is clear to know from the Fig.6 that K1 amplitude has a tendency to go increasing first and then decreasing from open sea to upstream which is similar to M2 component tide, but $\mathrm{K} 1$ amplitude is far less than M2. The attenuation of $\mathrm{K} 1$ amplitude is slower than M2 from open sea to upstream, and the difference between M2 and K1 amplitudes is only $0.05 \mathrm{~m}$ in the nearby Nanjing river reach.

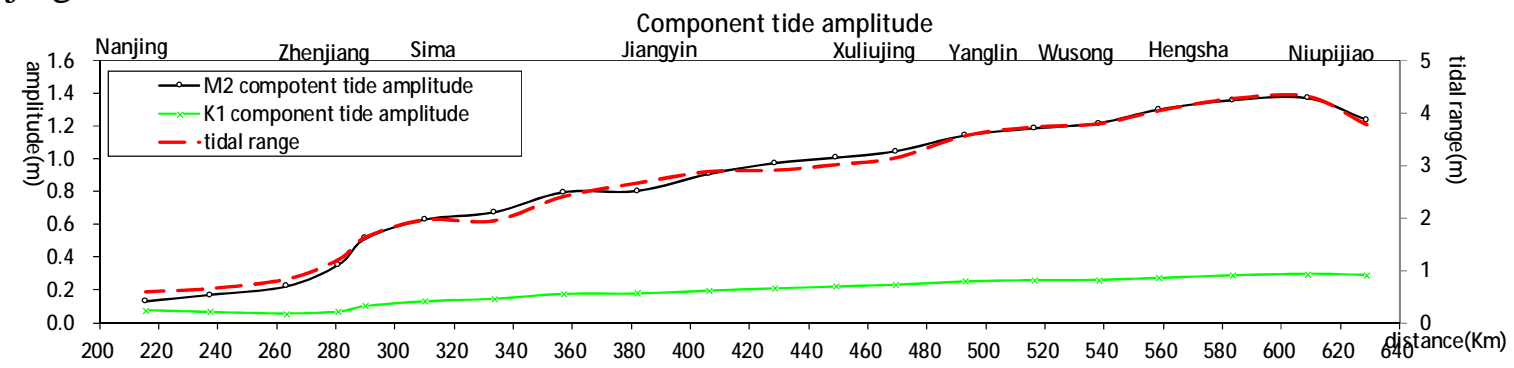

Fig.6 Amplitude of component tides

\section{Relationship between tidal level and tidal current}

Fig.7 shows the relationship between tidal level and tidal current of each tide station in Yangtze estuary. In open sea, the tidal wave is advancing wave whose phases of tidal level and tidal current are consistent with each other, that means, the maximum tidal current velocity occurs at high tidal level or low tidal level, while the minimum tidal current velocity occurs at average tidal level. In the semi-enclosed ecronic, the incident wave and reflected wave are superposed together to form a standing wave as a result of tidal wave reflection, besides, the phase difference between tidal level $\pi / 2$

and tidal current is $\pi / 2$, in other words, the tidal current velocity is minimum at high tidal level and low tidal level, while the tidal current velocity is maximum at average tidal level. In estuary, the tidal wave is mainly in the form of mixed wave, a combination of advancing wave and standing wave, which is different from open sea or semi-enclosed ecronic.

From Fig.7, the tidal wave, in Niupijiao station, is a mixed wave while the advancing wave is dominant for the tidal wave reflecting causing by Yangtze estuary Deepwater Channel Regulation Project. When the tidal wave approaches to Hengsha station, the property of advancing wave is strengthened for the channel is straight with little tidal wave reflection. The property of tidal wave in Wusong is similar to Hengsha station. When the tidal wave approaches to Xuliujing station, the maximum tidal current velocity occurs at high tidal level and low tidal level, and the phases of tidal level and tidal current are consistent with each other, so the tidal wave is manifestly the advancing wave. When the tidal wave propagates to Jiangyin station, the tidal current is mainly ebb current with little flood current, and the tidal wave is advancing wave which is similar to Xuliujing station. The Zhenjiang station is located in the upstream of tidal current limit whose tidal level gets influenced by tidal wave but the direction of flow is single to downstream, and the discharge has nothing to do with tidal wave, that is to say, the tidal current velocity increases while tidal level cutting down, on the contrary, the tidal current velocity decreases while tidal level rising.

Above all, when the tidal wave propagates from Nipijiao station to tidal current limit (near Jiangyin station), the property of advancing wave is gradually strengthened. 

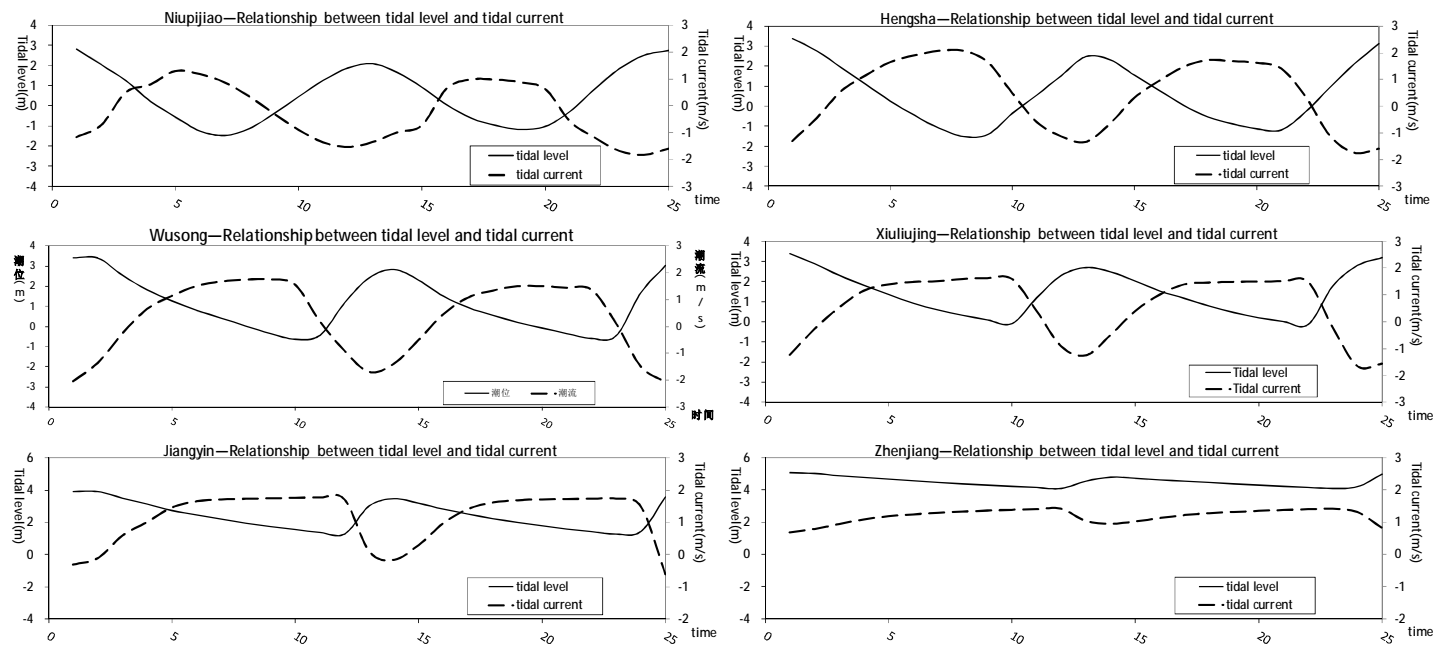

Fig.7 Relationship between tidal level and tidal current in Yangtze estuary

\section{Conclusions}

(1) For the tidal wave energy accumulation causing by the boundary shape contraction of Yangtze estuary and the tidal wave refection causing by Yangtze estuary Deepwater Channel Regulation Project in the nearby Niupijiao station, the high tidal level gets higher, low tidal level gets lower, and the tidal range increases. As the tidal wave propagates to upstream, both of the high tide and low tide get higher causing by runoff resistance and riverbed bottom friction. The tidal range from Niupijiao to upstream decreases generally, but increases slightly for tidal wave reflection near Sima.

(2) Semidiurnal component tide is dominant in Yangtze estuary, and the changing of M2 amplitude coincides well with the changing of tidal range.

(3) From the analysis of relationship between tidal level and tidal current, the property of advancing wave is strengthened gradually from Niupijiao station to tidal current limit(near Jiangyin).

\section{References}

[1].Dongsheng Yu. Analysis on Impact of the Coriolis Forces on Flow in the Yangtze River Estuary[C]. Third Chinese-German Joint Symposium on Coastal and Ocean Engineering, National Cheng Kung University, Tainan, 2006: 8-16.

[2].Lei Zhiyi, Zhang Jinshan, Kong Jun. Numerical Simulation of Water Level Under Interaction Between Runoff and Estuarine DynallHcs in Tidal Reach of the Yangtze River [J]. China Ocean Engineering Society, 2009, Vol.23, No.3: 543-551.

[3].Hanxing $\mathrm{Xu}$. Yangtze estuarine tidal variation and its engineering application [J]. Oceanologia et Limnologia Sinica, 1990, Vol.21, No.4: 311-319.

[4]. Zhichang Chen, Xiaofeng Luo. Summary of findings of Yangtze Estuary Deepwater Channel Regulation Project physical model tests [J]. Port and Waterway Engineering, 2006, vol.12: 134-140.

[5]. Huanting Shen. Propagation Yangtze Estuarine tidal wave and its impact on the evolution of channel [M]. Beijing: Science Press, 1981.

[6]. Yixin Yan, Junwei Liu, etc. Analysis of Yangtze Estuarine tidal characteristics before and after comprehensive renovation project [J]. Journal of Hohai University, 2009, Vol.37, No.1: 100-104.

[7].Dongsheng Yu, Chun Tian, etc. Analysis of Yangtze Estuarine flow moving features [J]. Port and Waterway Engineering, 2004, Vol.360, No.1: 49-53.

[8].Zhengdong Yang, Jianrong Zhu, Biao Wang, Tangyu Lin. Analysis on tides characteristics of tide stops in Yangtze estuary [J]. Journal of East China Normal University (Natural Science Edition), 2012, vol.3: 111-119.

[9]. Chuanteng Lu, Zhichang Chen, Xiaofeng Luo. The 2D and 3D nested tidal numerical simulation of Yangtze Estuary with FVM [J]. Hydro-Science and Engineering. 2013, 4: 18-23. 